\title{
Effects of Macroeconomic Uncertainty on the Stock and Bond Markets*
}

\author{
Hossein Asgharian, Lund University ${ }^{+}$ \\ Charlotte Christiansen, CREATES, Aarhus University ${ }^{++}$ \\ and \\ Ai Jun Hou, Stockholm University ${ }^{++}$
}

March 25, 2015

* Asgharian thanks the Jan Wallanders and Tom Hedelius Foundation for funding his research. Christiansen acknowledges financial support from The Danish Council of Independent Research (DFF - 4003-00022) and CREATES (Center for Research in Econometric Analysis of Time Series) funded by the Danish National Research Foundation (DNRF78).

+ Hossein Asgharian, Department of Economics, Lund University, Box 7082, 22007 Lund, Sweden. Hossein.Asgharian@nek.lu.se

++ Charlotte Christiansen, CREATES, Department of Economics and Business, School of Business and Social Sciences, Aarhus University, Fuglesangs Allé 4, 8210 Aarhus V, Denmark. cchristiansen@creates.au.dk.

+++ Ai Jun Hou, School of Business, Stockholm University, Sweden. ajh@fek.su.se. 


\title{
Effects of Macroeconomic Uncertainty on the Stock and Bond Markets
}

\begin{abstract}
In this paper we show that the long-run stock and bond volatility and the long-run stockbond correlation depend on macroeconomic uncertainty. We use the mixed data sampling (MIDAS) econometric approach. The findings are in accordance with the flight-to-quality phenomenon when macroeconomic uncertainty is high.
\end{abstract}

Keywords: DCC-MIDAS model; GARCH-MIDAS model; Macroeconomic uncertainty index; Stock-bond correlation; Stock volatility; Bond volatility

JEL Classifications: C32; C58; E32; E44; G11; G12 


\section{Introduction}

In this paper we investigate how the long-run volatility and correlation of stocks and bonds are influenced by macroeconomic uncertainty. We apply the macroeconomic uncertainty index (MUI) recently developed by Bali, Brown, and Tang (2014). The macroeconomic uncertainty index is based on the dispersion in survey forecasts for various macroeconomic variables. We apply the mixed data sampling (MIDAS) approach to combine the quarterly MUI with the daily stock and bond returns. This particular approach allows us to investigate the effect of macroeconomic uncertainty on the long-run stock and bond characteristics. To begin with, we consider univariate specifications for the conditional stock and bond volatility. Subsequently, we investigate the conditional stock-bond correlation. We find that macroeconomic uncertainty holds significant influence on the long-run stock volatility and the long-run stock-bond correlation, while the longrun bond volatility is unaffected. The long-run correlation tends to be small/negative when macroeconomic uncertainty is high. This finding is in accordance with the flight-to-quality phenomenon, i.e. the transfer of money from the high-risk stock market to the low-risk bond market in bad times.

The previous literature documents that the stock and bond comovement is influenced by various macro-finance variables, e.g. Viceira (2012) and Asgharian, et al. (2014). However, the current paper is the first to investigate the effects of macroeconomic uncertainty on the stock-bond comovement. Bali et al. (2014) find that the MUI explains a significant proportion of the crosssectional dispersion in stock returns.

The structure of the remaining part of the paper is as follows. Firstly, we present the data and methodology in section 2. Subsequently, we show the in-sample results for long-run volatility (section 3) and long-run correlation (section 4). Then we provide out-of-sample analysis before we conclude. 


\section{Data and Methodology}

The sample period is from 1986Q1 to 2014Q2. We use the daily returns from the settlement prices of the S\&P500 futures contract traded at the CME and the 10-year Treasury note futures contract traded at the CBT (both series are available from DataStream).

Following Bali et al. (2014), we construct the macroeconomic uncertainty index (MUI) as the first principal component of seven cross-sectional dispersion variables for the current quarter forecasts from the Survey of Professional Forecasters (SPF) database at the Federal Reserve Bank of Philadelphia (real GDP growth and level, nominal GDP growth and level, GDP price index growth and level, and the unemployment rate). The MUI explains $55 \%$ of the variation in the seven underlying series.

We investigate the effects of macroeconomic uncertainty on the long-run stock (and bond) volatility using the GARCH-MIDAS model of Engle, Ghysels, and Sohn (2013). We allow the long-run variance to depend on the lagged MUI, similar to how Asgharian, et al. (2013) account for influence from an exogenous variable on the stock volatility.

The stock return at day $i=1, . . N_{t}$ in quarter $t$ follows the process: $r_{i, t}=\mu+\sqrt{\tau_{t} g_{i, t}} \varepsilon_{i, t}$, where $\varepsilon_{i, t} \mid \Phi_{i-1, t} \sim N(0,1)$ and $\Phi_{i-1, t}$ is the information set up to day (i-1) of period $t$. The total variance is divided into a short-run component $g_{i, t}$ and a long-run component $\tau_{t}$ :

$$
\sigma_{i t}^{2}=\tau_{t} g_{i, t}
$$

The short-run component follows a GARCH $(1,1)$ process: $g_{i, t}=(1-\alpha-\beta)+\alpha \frac{\left(r_{i-1, t}-\mu\right)^{2}}{\tau_{t}}+\beta g_{i-1, t}$ where $\alpha>0$ and $\beta \geq 0, \alpha+\beta<1$. The long-run component is the smoothed realized volatility in a MIDAS regression where $M U I_{t-k}$ enters directly ( $K=12$ in this paper):

$$
\log \left(\tau_{t}\right)=\theta_{0}+\theta_{1} \sum_{k=1}^{K} \varphi_{k}\left(w_{1}, w_{2}\right) M U I_{t-k}
$$


We use a fixed window which means that the component $\tau_{t}$ does not change within period $t$. The weighting scheme used in eq. (2) is described by a beta lag polynomial, $\varphi_{k}\left(w_{1}, w_{2}\right)=\frac{(k / K)^{w_{1}-1}(1-k / K)^{w_{2}-1}}{\sum_{j=1}^{K}(j / K)^{w_{1}-1}(1-j / K)^{w_{2}-1}}, \quad k=1, \ldots ., 12$. We fix $w_{1}=1$ so the weighting scheme guarantees a decaying pattern where the rate to decay is determined by $w_{2}$. The parameter $\theta_{1}$ measures the effect of MUI on the long-run volatility.

The bivariate DCC-MIDAS model of Colacito, Engle, and Ghysels (2011) is a natural extension and combination of the DCC model of Engle (2002) and the GARCH-MIDAS model. Using this model, we allow the long-run correlation to depend directly on the lagged macroeconomic uncertainty, similarly to how Conrad, Loch, and Rittler (2014) and Asgharian et al. (2014) allow for influence from an exogenous variable in the DCC-MIDAS model.

The return vector follows the process: $r_{t} \sim N\left(\mu, H_{t}\right)$ and the conditional covariance matrix is specified as $H_{t}=D_{t} R_{t} D_{t}$, where $D_{t}$ is a diagonal matrix with the conditional volatilities and $R_{t}$ is the conditional correlation matrix of the standardized return residuals ( $\xi_{S, k}$ and $\xi_{B, k}$ ) from the univariate GARCH-MIDAS models (estimated in the first step). In the second step, their conditional covariance is estimated. The conditional correlation is given as $R_{t}=\operatorname{diag}\left(Q_{t}\right)^{-1 / 2} Q_{t} \operatorname{diag}\left(Q_{t}\right)^{-1 / 2} . \quad q_{t} \quad$ is the short-run correlation, $q_{t}=\bar{\rho}_{t}(1-a-b)+a \xi_{S, t-1} \xi_{B, t-1}+b q_{t-1}$ and $\bar{\rho}_{t}$ is a slowly moving long-run correlation: $\bar{\rho}_{t}=\frac{\exp \left(2 \bar{z}_{\tau}\right)-1}{\exp \left(2 \bar{z}_{\tau}\right)+1}$ where

$$
\bar{z}_{\tau}=\gamma_{0}+\gamma_{1} \sum_{k=1}^{K} \delta_{k}\left(w_{1}, w_{2}\right) M U I_{t-k}, k=1, \ldots . ., 16
$$


$\rho_{t}$ is the conditional correlation between the standardized residuals: $\rho_{t}=\frac{q_{t}}{\sqrt{q_{s, t} q_{B, t}}}$. The weithting scheme $\delta_{k}\left(w_{1}, w_{2}\right)$ is defined similarly to $\varphi_{k}\left(w_{1}, w_{2}\right)$. The parameter $\gamma_{1}$ measures the effect of the MUI on the long-run stock-bond correlation.

\section{Macroeconomic Uncertainty and the Long-Run Volatility}

Panel A (first part) of Table 1 shows the results from estimating the GARCH-MIDAS model for the stock returns when we use MUI in the MIDAS equation. We find that $\hat{\theta}_{1}$ is positive and significant for the stock returns. Firstly, this implies that the macroeconomic uncertainty has a significant bearing on the long-run stock volatility. Secondly, this implies that the higher the macroeconomic uncertainty is, the larger is the long-run stock volatility. The first diagram of Figure 1 shows the total and the long-run stock volatility. The long-run volatility is much smoother than the total volatility but it still follows the same general pattern. The macroeconomic uncertainty is very large during the recession period in the beginning of the 1990s, the burst of the IT bubble in 2001-2002 and the financial crisis in 2008-2009. This pattern is shared by the long-term component of the stock return volatility. The total volatility is also high in the periods with large macroeconomic uncertainty.

We also estimate the GARCH-MIDAS model for rolling window sub-samples starting with 1989Q1-2003Q4 and then moving the estimation window one year forward each time. $\hat{\theta}_{1}$ is not significant in the earlier part of the periods. Thus, it is the most recent observations that make the macroeconomic uncertainty effect significant for the entire sample period. The results are not reported but are available on request. 
The effect from MUI on the long-run bond volatility is negative but insignificant, cf. Panel A of Table 1. Unlike stocks, the long-run bond volatility is close to constant. As we see in the second diagram of Figure 1, during the financial crisis of 2008-2009 both macroeconomic uncertainty and total bond return volatility are very large, but such a clear pattern cannot be observed in other parts of the sample. This motivates the poor/flat estimation of the long-run component of the bond return volatility.

The estimations with rolling window sub-samples show that in the beginning the effect of the macroeconomic uncertainty on the long-run bond volatility is negative and insignificant and then it turns positive and significant in the last part of the sample (results available on request). Thus, it is the earliest observations that make the effect of the macroeconomic uncertainty on the long-run bond volatility insignificant.

\section{Macroeconomic Uncertainty and the Long-Run Correlation}

Panel B of Table 1 shows the results from estimating the DCC-MIDAS model. The parameter estimate of the MUI dependence is negative and significant; $\hat{\gamma}_{1}<0$. Thus, macroeconomic uncertianty has a significant effect on the long-run stock-bond correlation.

The negative sign of the MUI parameter estimate implies that when the macroeocnomic uncertainty is large, the long-run stock-bond correlation tends to be small, i.e. negative. Similarly, when the macroeconomic uncertainty is small, the long-run stock-bond correlation tends to be large, i.e. positive. This finding is consistent with the flight-to-quality phenomenon taking place when the macroeconomic uncertainty is large.

The third diagram of Figure 1 shows the long-run stock-bond correlation. Similar to the long-run stock volatility, the long-run stock-bond correlation is much smoother than the total correlation. The long-run correlation is both positive and negative during the sample period. The long-run 
correlation is negative in the same periods as the long-run stock volatility is large; namely in the early 1990s, in the early 2000s, and during the recent financial crisis. Thus, our findings suggest that the long-run stock-bond correlation changes sign depending on the macroeconomic conditions.

The rolling window estimation of the MUI dependence of the correlation is always significantly negative and slightly time varying.

\section{Comparison to GDP Growth Rate}

For robustness we compare the long-run effects from macroeocnomic uncertainty to those from the GDP growth rate, see Table 1. The GDP growth rate is a popular measure of the state of the economy. The correlation between the macroeonomic uncertainty index and the GDP growth rate is negative and very low, -0.042 . Thus, it is not the case that large macroeocnomic uncertainty is present at the same time as the GCP growth rate is low. The low correlation of the two variables indicates that they contain different information. The MUI has the advantage over the GDP that it is timely available and not subject to change.

For the long-run stock volatility, the GDP effect is positive (similar to MUI) but only boarderline signifnicant. This implies that when the GDP growth rate is large the long-run stock volatility tends to be large. Thus, the effect on the long-run stock volatility of large macroeconomic uncertainty is similar to the effect of large GDP growth rate. Neither MUI nor the GDP has a significant effect on the long-run bond volatility.

For the long-run correlation, the $\gamma_{1}$ parameter estimate is significantly negative for the MUI and significantly positive for the GDP grwoth rate. This means that large GDP growth implies positive long-run stock-bond correaltion, and small GDP growth implies negative long-run stock-bond correlaiton. Thus, when the macroeconomy is troubled there tends to be a negative correlaiton between stocks and bonds. This is in accordance with flight-to-quality behaviour. 
Overall, the macroeconomic uncertainty and GDP growth rates provide similar information about the type of macroeconomic dependence of long-run stock and bond volatility and long-run stockbond correlation.

\section{Out-of-Sample Analysis}

We use the rolling window estimation from above to provide out-of-sample estimates four quarters into the future based on the MUI and the GDP specification. For comparison, we also consider the random walk specification.

In Table 2, we provide the mean squared error (MSE) from this. The MSE is smallest for the random walk specification (bond volatility and stock-bond correlation) or the MUI specificaiton (stock volatility). Yet, the differences in MSE values are fairly small and do not allow us to draw unambigious strong conclusions with respect to the strenght of the out-of-sample results. Table 2 also shows the standard deviation of the minimum variance portfolios; first for the unrestricted minimum variance portfolio, second for the restriction of identical variance across models, and third with imposed identical correlation across models. Here, the specification with macroeconomic uncertainty is preferred over the random walk which is preferred over the GDP.

Engle and Colacito (2006) propose a new way of comparing the out-of-sample performance of correlation models, namely a test statistic that provides pairwise comparisons of covariance matrices corresponding to the minimum variance portfolios for any given expected return. Table 3 holds the corresponding Engle-Colacito test statistics. For various levels of expected returns for stock and bonds, the MUI specification is always better than or equal to the GDP specification, and the same applies when comparing the MUI with the random walk. For the joint test across all return levels neither of the specifications is significantly better than the others. 
Overall, for the long-run stock and bond volatility and the long-run stock-bond correlation the outof-sample analysis does not convincingly document a strong role for the state of the macro economy. This is much surprising giving the strong in-sample results.

\section{Conclusion}

In this paper we show how macroeconomic uncertainty influences the long-run stock and bond volatility and the long-run stock-bond correlation using the MIDAS approach. The effect of macroeconomic uncertainty is increasing over time. The empirical results show flight-to-quality behavior. The effects from the alternative macroeconomic state variable, the GDP growth rate, are similar to the effects from macroeconomic uncertainty. The out-of-sample results are fairly weak with respect to the influence of the macroeconomic uncertainty.

\section{References}

Asgharian, H., A.J. Hou, and F. Javed (2013). Importance of the macroeconomic variables for variance prediction A GARCH-MIDAS approach. Journal of Forecasting, Forthcoming.

Asgharian, H., C. Christiansen, and A.J. Hou (2014). Macro-Finance Determinants of the Long-Run Stock-Bond Correlation: The DCC-MIDAS Specification. Working Paper SSRN.

Bali, T.G, S.J. Brown, and Y. Tang (2014): Macroeconomic Uncertainty and Expected Stock Returns. Working Paper SSRN.

Colacito, R., R.F. Engle, and E. Ghysels (2011). A Component Model for Dynamic Correlations. Journal of Econometrics 164, 45-59.

Conrad, C., K. Loch, and D. Ritter (2014). On the Macroeconomic Determinants of the Long-Term Oil-Stock Correlation. Journal of Empirical Finance 29, 26-40. 
Engle R, E. and R. Colacito (2006). Testing and Valuing Dynamic Correlations for Asset Allocation. Journal of Business and Economic Statistics 24, 238-253

Engle R, E. Ghysels, and B. Sohn (2013). On the Economic Sources of Stock Market Volatility. Review of Economics and Statistics 93, 776-797.

Viceira, L.M. (2012). Bond Risk, Bond Return Volatility, and the Term Structure of Interest Rates. International Journal of Forecasting 28, 97-117. 


\section{Table 1: In-Sample Parameter Estimates}

Panel A of the table reports the results of the univariate GARCH-MIDAS model, where the longrun variance is defined as the function of the macroeconomic uncertainty index (MUI) or the GDP growth rate. $\mu$ is the intercept term in the mean equation for returns, $\alpha$ and $\beta$ are the parameters of the short term variance, $w_{2}$ is the estimated weight (smoothing) parameter, $\theta_{0}$ is the intercept term in the long-run variance equation, and $\theta_{1}$ is the estimated parameter of MUI/GDP.

Panel B of the table reports the results of the bivariate DCC-MIDAS model for stocks and bonds, where the long-run correlation is defined as the function of the macroeconomic uncertainty index (MUI) or the GDP growth rate. $a$ and $b$ are the parameters of the short term correlation, $\gamma_{0}$ is the intercept term in the long-run correlation equation, $\gamma_{1}$ is the estimated parameter of MUI/GDP and $w_{2}$ is the estimated weight parameter.

The standard errors are given in italic below the estimated parameters and ${ }^{* * *},{ }^{* *}$ and * indicate significance at the $1 \%, 5 \%$ and $10 \%$ levels, respectively.

Panel A: GARCH-MIDAS Model

\begin{tabular}{|c|c|c|c|c|c|c|c|}
\hline & & $\mu$ & $\alpha$ & $\beta$ & $\theta_{0}$ & $\theta_{1}$ & $w_{2}$ \\
\hline \multirow{4}{*}{$M U I$} & Stocks & $0.0084^{* * *}$ & $0.0700^{* * *}$ & $0.9177^{* * *}$ & $-3.5921^{* * *}$ & $0.1668^{* * *}$ & $3.9571^{* *}$ \\
\hline & & 0.0015 & 0.0024 & 0.0014 & 0.1363 & 0.0426 & 1.9137 \\
\hline & Bonds & 0.0011 & $0.0373^{* * *}$ & $0.9529^{* * *}$ & $-5.2676^{* * *}$ & -0.1319 & $1.0000^{* * *}$ \\
\hline & & 0.0008 & 0.0019 & 0.0018 & 0.0921 & 0.1385 & 0.0002 \\
\hline \multirow{4}{*}{$G D P$} & Stocks & 0.0011 & $0.0374^{* * *}$ & $0.9532^{* * *}$ & $-5.2605^{* * *}$ & 0.0469 & 1.2963 \\
\hline & & 0.0008 & 0.0018 & 0.0017 & 0.0957 & 0.0485 & 1.3526 \\
\hline & Bonds & $0.0083^{* * * *}$ & $0.0848^{* * * *}$ & $0.8984^{* * *}$ & $-3.7241^{* * *}$ & $0.3498^{*}$ & $1.0000^{* * * *}$ \\
\hline & & 0.0015 & 0.0025 & 0.0019 & 0.1043 & 0.1806 & 0.0002 \\
\hline
\end{tabular}

Panel B: DCC-MIDAS Model

\begin{tabular}{lccccc}
\hline & $a$ & $b$ & $\gamma_{0}$ & $\gamma_{1}$ & $w_{2}$ \\
\hline MUI & $0.0372^{* * * *}$ & $0.9578^{* * * *}$ & -0.0977 & $-0.1684^{* * *}$ & 2.9710 \\
& 0.0010 & 0.0012 & 0.0890 & 0.0797 & 2.2930 \\
GDP & $0.0376^{* * * *}$ & $0.9572^{* * * *}$ & -0.0244 & $0.3855^{* * *}$ & $1.4595^{* *}$ \\
& 0.0010 & 0.0012 & 0.0829 & 0.1638 & 0.8045 \\
\hline
\end{tabular}




\section{Table 2: Out-of-Sample Results}

The table shows the out-of-sample results of the bivariate DCC-MIDAS model for stocks and bonds, where the long-run correlation is defined as the function of the macroeconomic uncertainty index (MUI) or the GDP growth. The estimates are updated each year using a rolling window of four year. The out-of-sample performance of the model is compared with that of a random walk (RW) forecast method using one lag realized variances and correlation. The first part of the table compares the computed mean square error (MSE) of the comparison of the predicted standard deviations and correlation with the corresponding realized values. The second part of the table shows the standard deviations of the minimum variance portfolio (MVP) where the portfolio weights are obtained using from the three alternative estimations. In the second (third) row of this part of the table we use the variance (covariance) estimated for DCC-MIDAS model with MUI for other models.

\begin{tabular}{llccc}
\hline & & MUI & GDP & RW \\
\hline \multirow{3}{*}{ MSE } & Variance Stocks & 0.0052 & 0.0059 & 0.0055 \\
& Variance Bonds $(\times 100)$ & 0.0023 & 0.0018 & 0.0011 \\
& Correlation & 0.1150 & 0.1370 & 0.0778 \\
\hline \multirow{3}{*}{ Std of MVP } & Same variance & 0.1238 & 0.1318 & 0.1271 \\
& Same covariance & 0.1238 & 0.1287 & 0.1238 \\
& 0.1238 & 0.1264 & 0.1265 \\
\hline
\end{tabular}

Table 3: $t$-Values from Engle-Colacito (2006) test

The table shows the t-values from Engle-Colacito (2006) test. A negative $t$-value shows that the first model is better than the second model, i.e. it gives a lower variance for the given level of the expected return. The significant $t$-values at $5 \%$ level are marked with bold.

\begin{tabular}{|c|c|c|c|c|c|c|c|}
\hline \multicolumn{2}{|c|}{ Expected return } & \multicolumn{3}{|c|}{ Unweighted test } & \multicolumn{3}{|c|}{ Weighted test } \\
\hline Stocks & Bonds & $\begin{array}{c}\text { MUI- } \\
\text { GDP }\end{array}$ & $\begin{array}{c}M U I- \\
R W\end{array}$ & $\begin{array}{c}\text { GDP- } \\
R W\end{array}$ & $\begin{array}{l}M U I- \\
G D P\end{array}$ & $\begin{array}{c}M U I- \\
R W\end{array}$ & $\begin{array}{r}G D P- \\
R W \\
\end{array}$ \\
\hline 0 & 1 & 1,33 & 0,79 & 0,58 & 1,91 & 0,98 & 1,38 \\
\hline 0,16 & 0,99 & $-1,18$ & $-0,81$ & $-0,74$ & $-2,20$ & $-1,71$ & $-1,17$ \\
\hline 0,31 & 0,95 & 1,20 & 1,27 & $-0,15$ & 0,51 & 1,52 & $-1,14$ \\
\hline 0,45 & 0,89 & $-2,88$ & 1,90 & 0,82 & $-1,39$ & $-1,43$ & 0,52 \\
\hline 0,59 & 0,81 & $-0,24$ & 0,84 & 2,19 & $-0,63$ & $-0,47$ & 3,08 \\
\hline 0,71 & 0,71 & 1,48 & 0,13 & 0,87 & 0,13 & $-0,61$ & 1,44 \\
\hline 0,81 & 0,59 & $-0,38$ & 1,90 & $-0,12$ & $-0,78$ & $-0,60$ & $-1,34$ \\
\hline 0,89 & 0,45 & $-0,70$ & 0,07 & $-1,24$ & 0,69 & 0,60 & $-0,67$ \\
\hline 0,95 & 0,31 & 0,36 & $-2,85$ & $-0,79$ & $-0,11$ & 0,05 & $-0,45$ \\
\hline 0,99 & 0,16 & $-3,55$ & $-1,92$ & $-0,62$ & $-4,57$ & $-3,98$ & $-0,27$ \\
\hline 1 & 0 & 0,94 & 1,10 & 1,59 & 2,34 & 3,17 & 1,20 \\
\hline \multicolumn{2}{|c|}{ Joint test } & $-0,14$ & $-0,05$ & 0,01 & $-0,14$ & $-0,12$ & 0,08 \\
\hline
\end{tabular}




\section{Figure 1: Estimated Return Volatilities and Correlation for Stocks and Bonds}

The figures plot the estimated return volatility (square root of the variance) and correlation for stocks and bonds, from the univariate GARCH-MIDAS and the bivariate DCC-MIDAS. The figure also presents the corresponding estimates of the long-run components of volatility and correlation. The long run components are defined as function of the macroeconomic uncertainty index (MUI) in the MIDAS equation. The figures also plot the quarterly values of MUI.

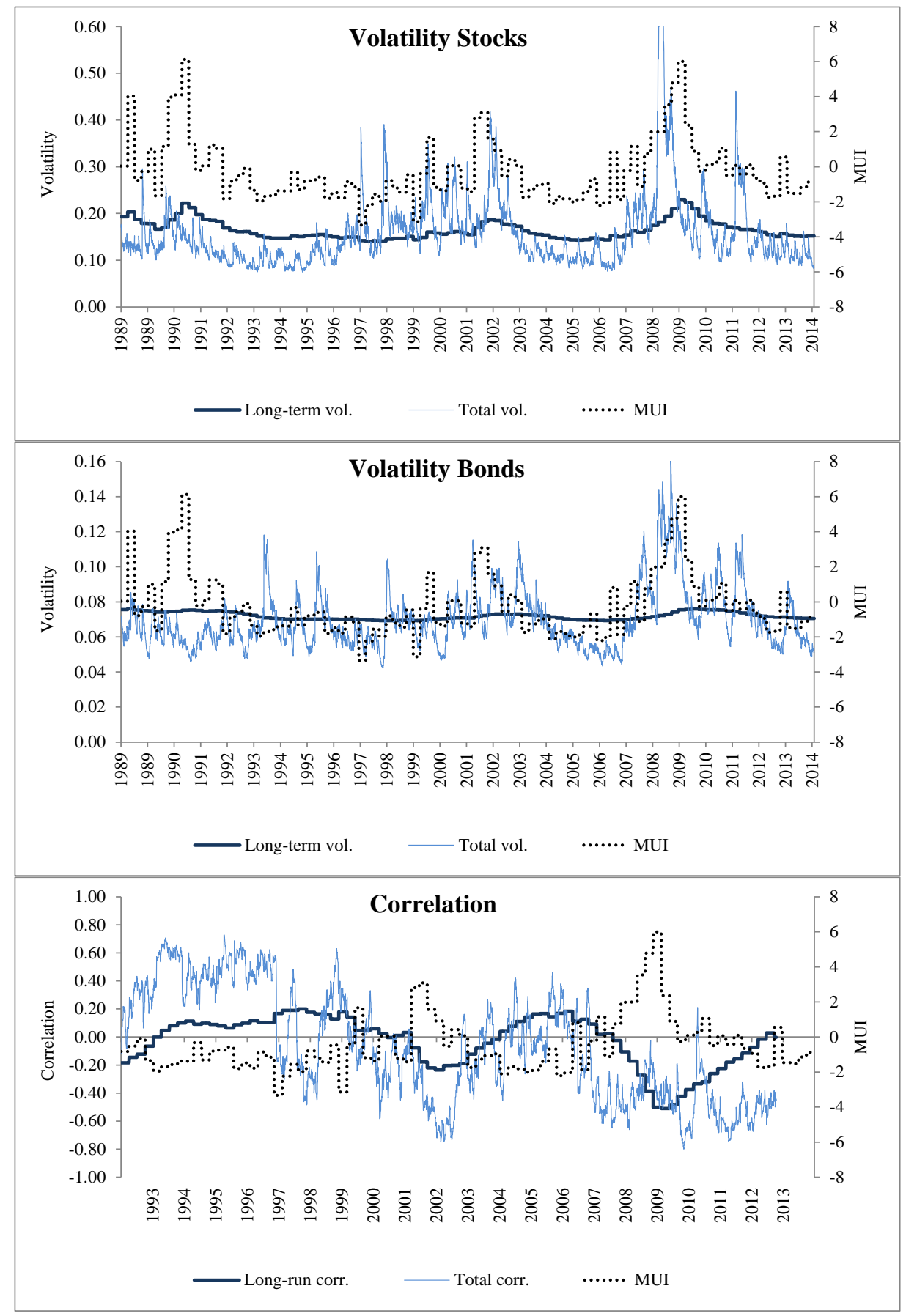

